\title{
A discrete negative AKNS equation: generalized Cauchy matrix approach
}

Song-lin Zhao

To cite this article: Song-lin Zhao (2016) A discrete negative AKNS equation: generalized Cauchy matrix approach, Journal of Nonlinear Mathematical Physics 23:4, 544-562, DOI: https://doi.org/10.1080/14029251.2016.1237201

To link to this article: https://doi.org/10.1080/14029251.2016.1237201

Published online: 04 January 2021 


\title{
A discrete negative AKNS equation: generalized Cauchy matrix approach
}

\author{
Song-lin Zhao \\ Department of Applied Mathematics, Zhejiang University of Technology \\ Hangzhou, 310023, Zhejiang, P.R. China \\ songlinzhao@zjut.edu.cn
}

Received 19 June 2016

Accepted 26 August 2016

\begin{abstract}
Generalized Cauchy matrix approach is used to investigate a discrete negative Ablowitz-Kaup-Newell-Segur (AKNS) equation. Several kinds of solutions more than multi-soliton solutions to this equation are derived by solving determining equation set. Furthermore, applying an appropriate continuum limit we obtain a semidiscrete negative AKNS equation and after a second continuum limit we derive the nonlinear negative AKNS equation. The reductions to discrete, semi-discrete and continuous sine-Gordon equations are also discussed.
\end{abstract}

Keywords: discrete negative AKNS equation; solution; continuum limit; reduction.

2000 Mathematics Subject Classification: 39A14, 35Q51, 37K40

\section{Introduction}

The negative order equation has been a major research target for many decades. A well-known example of the negative order equations is the sine-Gordon (SG) equation which can be derived as a negative order member of the modified Korteweg-de Vries (KdV) equation [23]. Generally speaking, for the positive hierarchy

$$
u_{t}=T^{n} K_{0}(u)=K_{n}, n=0,1, \cdots,
$$

where members are related through the recursion operator $T$, by the negative order hierarchy we refer to

$$
u_{t}=T^{-n} K_{0}(u), n=1,2, \cdots,
$$

i.e., the power of $T$ goes to the opposite direction. If the recursion operator is hereditary and admits an implectic-symplectic separation [12], the negative order equations also have multi-Hamiltonian structures [12]. Many physically meaningful systems, such as the Camassa-Holm equation [8], the Degasperis-Procesi equation [11], and the short pulse equation [25], are associated to negative order equations through reciprocal transformations (also see [17]). In [30], soliton solutions and asymptotic analysis are presented for a negative order AKNS (nAKNS) equation, which is actually the SG equation in non-potential form. It is found that two-soliton scattering provides not only phase shifts but also amplitude changes for each soliton, which differs from classical soliton interactions of which only phase shifts appear. Recently, Qiao and Fan systematically study two negative order $\mathrm{KdV}$ equations, particularly including Hamiltonian structures, Lax pairs, conservation laws, and explicit multisoliton and multikink wave solutions [22]. 
The discrete version of soliton system, i.e. system given by integrable partial difference equation, has been a popular topic and is drawing more and more attention. The study of integrable partial difference equations dates back to the pioneering work of Ablowitz and Ladik [1,2], and of Hirota [16], where integrable discretizations of AKNS equation, KdV equation, Toda chain, SG equation are proposed. Subsequently, Sato's approach [10] and direct linearisation method [19] are also established to construct the discrete integrable system. In [24], by introducing discrete plane wave factor, many discrete equations are proposed, including nonlinear Schrödinger equation, isotropic classical Heisenberg spin chain and complex and real SG equation. Besides, semidiscrete equations and continuous equations are also discussed by applying continuum limits. With the help of property of multi-dimensional consistency $[6,20]$, several discrete systems are classified, including Adler-Bobenko-Suris lattice list [3], lattice Boussinesq type equations [13] and lattice Kadomtsev-Petviashvili type equations [4]. With regard to the solutions for discrete integrable system, some approaches have been developed in recent years, such as Cauchy matrix approach [18], generalized Cauchy matrix approach [31], bilinear method [14], Bäcklund transformation [5], Darboux transformation [21], inverse scattering transformation [7] and algebro-geometric method [9].

In [28], Yu investigates two semi-discrete and one fully discrete versions of the nAKNS equation via Hirota's bilinear method and discusses soliton solutions and dynamics. In this paper, motivated by the generalized Cauchy matrix approach and the understanding discrete dispersion relation [15, 32 ], we study another discrete version of the nAKNS equation. The paper is organized as follows. In Sec.2, we start from the Sylvester equation (see (2.2)) and see that $\boldsymbol{S}^{(i, j)}$ (defined as (2.6)) obeys some properties, such as recurrence relations and invariance. These recurrence relations can be viewed as discrete equations of $\boldsymbol{S}^{(i, j)}$ with discrete independent variables $i, j$. In Sec.3, by imposing dispersion relations on $\boldsymbol{r}$, a discrete nAKNS equation is obtained in closed form. In Sec.4, we solve determining equation set and construct several kinds of solutions more than multi-soliton solutions. In Sec.5, we discuss continuum limits of the resulting discrete nAKNS equation. Sec.6 is devoted to the reduction. Section 7 is for conclusions. In addition, an appendix is given as a complement to the article.

\section{The Sylvester equation and master function}

In this section, the Sylvester equation is firstly introduced, which is always taken as the starting point of generalized Cauchy matrix approach. Moreover, matrix function $\boldsymbol{S}^{(i, j)}$ is given as well as some properties are discussed, such as recurrence relation and invariance.

Proposition 2.1. [26] Let us denote the eigenvalue sets of $\boldsymbol{X}$ and $\boldsymbol{Y}$ by $\mathscr{E}(\boldsymbol{X})$ and $\mathscr{E}(\boldsymbol{Y})$, respectively. For the known $\boldsymbol{X}, \boldsymbol{Y}$ and $\boldsymbol{Z}$, the Sylvester equation

$$
X M-M Y=Z
$$

has a unique solution $\boldsymbol{M}$ if and only if $\mathscr{E}(\boldsymbol{X}) \cap \mathscr{E}(\boldsymbol{Y})=\varnothing$.

\subsection{The Sylvester equation}

The Sylvester equation utilized in present paper is

$$
\boldsymbol{K M}-\boldsymbol{M K}=\boldsymbol{r}^{t} \boldsymbol{c},
$$


where matrices $\boldsymbol{K}, \boldsymbol{M}, \boldsymbol{r}$ and ${ }^{t} \boldsymbol{c}$ are of form

$$
\begin{aligned}
& \boldsymbol{K}=\left(\begin{array}{cc}
\boldsymbol{K}_{1} & \mathbf{0} \\
\mathbf{0} & \boldsymbol{K}_{2}
\end{array}\right), \quad \boldsymbol{M}=\left(\begin{array}{cc}
\mathbf{0} & \boldsymbol{M}_{1} \\
\boldsymbol{M}_{2} & \mathbf{0}
\end{array}\right), \\
& \boldsymbol{r}=\left(\begin{array}{cc}
\boldsymbol{r}_{1} & \mathbf{0} \\
\mathbf{0} & \boldsymbol{r}_{2}
\end{array}\right),{ }^{t} \boldsymbol{c}=\left(\begin{array}{cc}
\mathbf{0} & { }^{t} \boldsymbol{c}_{2} \\
{ }^{t} \boldsymbol{c}_{1} & \mathbf{0}
\end{array}\right)
\end{aligned}
$$

with $\boldsymbol{K}_{i} \in \mathbb{C}_{N_{i} \times N_{i}}, \boldsymbol{M}_{1} \in \mathbb{C}_{N_{1} \times N_{2}}, \boldsymbol{M}_{2} \in \mathbb{C}_{N_{2} \times N_{1}}, \boldsymbol{r}_{i} \in \mathbb{C}_{N_{i} \times 1},{ }^{t} \boldsymbol{c}_{i} \in \mathbb{C}_{1 \times N_{i}},(i=1,2)$ and $N_{1}+N_{2}=$ $2 N$. Here ${ }^{t} \boldsymbol{c}$ does not mean transpose of $\boldsymbol{c}$ but only a notation, transpose is represented by $T$. We suppose $\boldsymbol{r}_{i}=\left(r_{i, 1}, r_{i, 2}, \cdots, r_{i, N_{i}}\right)^{T}$ and ${ }^{t} \boldsymbol{c}_{i}=\left(c_{i, 1}, c_{i, 2}, \cdots, c_{i, N_{i}}\right),(i=1,2)$. The Sylvester equation (2.2) corresponds to $\boldsymbol{X}=\boldsymbol{Y}$ and $\boldsymbol{Z}$ being of rank 2 in (2.1). It is clear that the Sylvester equation (2.2) can be divided into the following system

$$
\begin{aligned}
& \boldsymbol{K}_{1} \boldsymbol{M}_{1}-\boldsymbol{M}_{1} \boldsymbol{K}_{2}=\boldsymbol{r}_{1}{ }^{t} \boldsymbol{c}_{2}, \\
& \boldsymbol{K}_{2} \boldsymbol{M}_{2}-\boldsymbol{M}_{2} \boldsymbol{K}_{1}=\boldsymbol{r}_{2}{ }^{t} \boldsymbol{c}_{1} .
\end{aligned}
$$

In terms of Proposition 2.1, we know that for arbitrary vectors $\boldsymbol{r}_{i},{ }^{t} \boldsymbol{c}_{i}(i=1,2)$, system (2.4) is solvable and has unique solution when $\mathscr{E}\left(\boldsymbol{K}_{1}\right) \cap \mathscr{E}\left(\boldsymbol{K}_{2}\right)=\varnothing$. In the rest parts of this section, we assume that $\boldsymbol{K}_{1}$ and $\boldsymbol{K}_{2}$ satisfy such condition. Besides, in order to avoid difficulties we also suppose $1 \notin \mathscr{E}\left(\boldsymbol{M}_{1} \boldsymbol{M}_{2}\right) \bigcup \mathscr{E}\left(\boldsymbol{M}_{2} \boldsymbol{M}_{1}\right)$ and $|\boldsymbol{K}| \neq 0$.

By using the Sylvester equation (2.2) repeatedly, we get the following result.

Proposition 2.2. For the matrix $\boldsymbol{M}$ defined by (2.2), we have the following relations

$$
\begin{aligned}
& \boldsymbol{K}^{s} \boldsymbol{M}-\boldsymbol{M} \boldsymbol{K}^{s}=\sum_{l=0}^{s-1} \boldsymbol{K}^{s-1-l} \boldsymbol{r}^{t} \boldsymbol{c} \boldsymbol{K}^{l}, \quad(s=1,2, \cdots), \\
& \boldsymbol{K}^{-s} \boldsymbol{M}-\boldsymbol{M} \boldsymbol{K}^{-s}=-\sum_{l=-1}^{-s} \boldsymbol{K}^{-s-1-l} \boldsymbol{r}^{t} \boldsymbol{c} \boldsymbol{K}^{l}, \quad(s=1,2, \cdots),
\end{aligned}
$$

where $\boldsymbol{K}^{0}=\boldsymbol{I}_{2 N}$ represents the 2 th-order unit matrix.

It is obvious that (2.5a) reduces to the Sylvester equation (2.2) when $s=1$. In the rest parts of present paper, we appoint that $\boldsymbol{I}_{j}$ indicates the $j$ th-order unit matrix. For convenience, we denote $\boldsymbol{I}_{2 N}=\boldsymbol{I}$.

\subsection{Master function $\boldsymbol{S}^{(i, j)}$}

\subsubsection{Definition of $\boldsymbol{S}^{(i, j)}$}

By the Sylvester equation (2.2) we introduce a $2 \times 2$ matrix function

$$
\boldsymbol{S}^{(i, j)}={ }^{t} \boldsymbol{c} \boldsymbol{K}^{j}(\boldsymbol{I}+\boldsymbol{M})^{-1} \boldsymbol{K}^{i} \boldsymbol{r}=\left(\begin{array}{ll}
s_{1} & s_{2} \\
s_{3} & s_{4}
\end{array}\right), i, j \in \mathbb{Z},
$$

together with a $2 N \times 2$ auxiliary matrix function

$$
\boldsymbol{u}^{(i)}=(\boldsymbol{I}+\boldsymbol{M})^{-1} \boldsymbol{K}^{i} \boldsymbol{r}, \quad i \in \mathbb{Z} .
$$

Similar to [27], here we call $\boldsymbol{S}^{(i, j)}$ the master function, since it will be used to yield integrable equations satisfied by $\boldsymbol{S}^{(i, j)}$. An apparent fact is

$$
\boldsymbol{S}^{(i, j)}={ }^{t} \boldsymbol{c} \boldsymbol{K}^{j} \boldsymbol{u}^{(i)}, \quad i, j \in \mathbb{Z} .
$$


In terms of the condition $1 \notin \mathscr{E}\left(\boldsymbol{M}_{1} \boldsymbol{M}_{2}\right) \cup \mathscr{E}\left(\boldsymbol{M}_{2} \boldsymbol{M}_{1}\right)$, we know that matrix $\boldsymbol{I}+\boldsymbol{M}$ is invertible. By direct calculation one can easily get the inverse of matrix $\boldsymbol{I}+\boldsymbol{M}$,

$$
(\boldsymbol{I}+\boldsymbol{M})^{-1}=\left(\begin{array}{cc}
\left(\boldsymbol{I}_{N_{1}}-\boldsymbol{M}_{1} \boldsymbol{M}_{2}\right)^{-1} & -\boldsymbol{M}_{1}\left(\boldsymbol{I}_{N_{2}}-\boldsymbol{M}_{2} \boldsymbol{M}_{1}\right)^{-1} \\
-\boldsymbol{M}_{2}\left(\boldsymbol{I}_{N_{1}}-\boldsymbol{M}_{1} \boldsymbol{M}_{2}\right)^{-1} & \left(\boldsymbol{I}_{N_{2}}-\boldsymbol{M}_{2} \boldsymbol{M}_{1}\right)^{-1}
\end{array}\right) .
$$

For the details of calculation, one can see Appendix A. Substituting (2.9) together with (2.3) into (2.6), we immediately arrive at the explicit expressions of the components $\left\{s_{i}\right\}$,

$$
\begin{aligned}
& s_{1}=-{ }^{t} \boldsymbol{c}_{2} \boldsymbol{K}_{2}^{j} \boldsymbol{M}_{2}\left(\boldsymbol{I}_{N_{1}}-\boldsymbol{M}_{1} \boldsymbol{M}_{2}\right)^{-1} \boldsymbol{K}_{1}^{i} \boldsymbol{r}_{1}, \\
& s_{2}={ }^{t} \boldsymbol{c}_{2} \boldsymbol{K}_{2}^{j}\left(\boldsymbol{I}_{N_{2}}-\boldsymbol{M}_{2} \boldsymbol{M}_{1}\right)^{-1} \boldsymbol{K}_{2}^{i} \boldsymbol{r}_{2}, \\
& s_{3}={ }^{t} \boldsymbol{c}_{1} \boldsymbol{K}_{1}^{j}\left(\boldsymbol{I}_{N_{1}}-\boldsymbol{M}_{1} \boldsymbol{M}_{2}\right)^{-1} \boldsymbol{K}_{1}^{i} \boldsymbol{r}_{1}, \\
& s_{4}=-{ }^{t} \boldsymbol{c}_{1} \boldsymbol{K}_{1}^{j} \boldsymbol{M}_{1}\left(\boldsymbol{I}_{N_{2}}-\boldsymbol{M}_{2} \boldsymbol{M}_{1}\right)^{-1} \boldsymbol{K}_{2}^{i} \boldsymbol{r}_{2} .
\end{aligned}
$$

\subsubsection{The recurrence relations}

Proposition 2.3. For the master function $\boldsymbol{S}^{(i, j)}$ defined by (2.6) with $\boldsymbol{M}, \boldsymbol{K}, \boldsymbol{r},{ }^{t} \boldsymbol{c}$ satisfying the Sylvester equation (2.2), we have the following relation

$$
\boldsymbol{S}^{(i, j+n)}=\boldsymbol{S}^{(i+n, j)}-\sum_{l=0}^{n-1} \boldsymbol{S}^{(n-1-l, j)} \boldsymbol{S}^{(i, l)}, \quad(n=1,2, \cdots) .
$$

In particular, when $n=1$ we have

$$
\boldsymbol{S}^{(i, j+1)}=\boldsymbol{S}^{(i+1, j)}-\boldsymbol{S}^{(0, j)} \boldsymbol{S}^{(i, 0)} .
$$

Proof. We consider the auxiliary matrix function $\boldsymbol{u}^{(i)}$, which is equivalent to

$$
(\boldsymbol{I}+\boldsymbol{M}) \boldsymbol{u}^{(i)}=\boldsymbol{K}^{i} \boldsymbol{r} .
$$

Multiplying (2.13) from the left by factor $\boldsymbol{K}^{n}$ and noting that relation (2.5a), we have

$$
(\boldsymbol{I}+\boldsymbol{M}) \boldsymbol{K}^{n} \boldsymbol{u}^{(i)}=\boldsymbol{K}^{n+i} \boldsymbol{r}+\sum_{l=0}^{n-1} \boldsymbol{K}^{n-1-l} \boldsymbol{r}^{t} \boldsymbol{c} \boldsymbol{K}^{l} \boldsymbol{u}^{(i)},
$$

which yields (2.11) after left-multiplying (2.14) by ${ }^{t} \boldsymbol{c} \boldsymbol{K}^{j}(\boldsymbol{I}+\boldsymbol{M})^{-1}$ and using the connection (2.8).

Proposition 2.4. For the master function $\boldsymbol{S}^{(i, j)}$ defined by (2.6) with $\boldsymbol{M}, \boldsymbol{K}, \boldsymbol{r},{ }^{t} \boldsymbol{c}$ satisfying the Sylvester equation (2.2), we have the following relation

$$
\boldsymbol{S}^{(i, j-n)}=\boldsymbol{S}^{(i-n, j)}+\sum_{l=-1}^{-n} \boldsymbol{S}^{(-n-1-l, j)} \boldsymbol{S}^{(i, l)}, \quad(n=1,2, \cdots) .
$$

In particular, when $n=1$ we have

$$
\boldsymbol{S}^{(i, j-1)}=\boldsymbol{S}^{(i-1, j)}+\boldsymbol{S}^{(-1, j)} \boldsymbol{S}^{(i,-1)} .
$$

The proof of this proposition is similar to the one for Proposition 2.3, which is omitted here. 


\subsubsection{Invariance of $\boldsymbol{S}^{(i, j)}$}

Suppose that under transform matrix $\boldsymbol{T}=\operatorname{Diag}\left(\boldsymbol{T}_{1}, \boldsymbol{T}_{2}\right)$, matrix $\overline{\boldsymbol{K}}$ is similar to $\boldsymbol{K}$, i.e.

$$
\bar{K}=\boldsymbol{T} K \boldsymbol{T}^{-1} \text {. }
$$

We denote

$$
\overline{\boldsymbol{M}}=\boldsymbol{T} \boldsymbol{M} \boldsymbol{T}^{-1}, \overline{\boldsymbol{r}}=\boldsymbol{T r},{ }^{\bar{c}} \boldsymbol{c}={ }^{t} \boldsymbol{c} \boldsymbol{T}^{-1} .
$$

Then one can easily know that

$$
\overline{\boldsymbol{K}} \overline{\boldsymbol{M}}-\overline{\boldsymbol{K}} \overline{\boldsymbol{M}}=\overline{\boldsymbol{r}}^{\bar{c}}
$$

and

$$
\boldsymbol{S}^{(i, j)}={ }^{t} \boldsymbol{c} \boldsymbol{K}^{j}(\boldsymbol{I}+\boldsymbol{M})^{-1} \boldsymbol{K}^{i} \boldsymbol{r}={ }^{\bar{c}} \boldsymbol{c} \overline{\boldsymbol{K}}^{j}(\boldsymbol{I}+\overline{\boldsymbol{M}})^{-1} \overline{\boldsymbol{K}}^{i} \overline{\boldsymbol{r}}
$$

(2.18b) means that $\boldsymbol{S}^{(i, j)}$ is invariant under the similar transformation (2.17).

In conclusion, we in this section introduce the Sylvester equation as well as the master function $\boldsymbol{S}^{(i, j)}$. By the Sylvester equation itself, we identify some properties of $\boldsymbol{S}^{(i, j)}$. In the next section, a discrete nAKNS equation is constructed. To proceed, we introduce two diagonal matrices

$$
\boldsymbol{A}=\left(\begin{array}{cc}
\boldsymbol{I}_{N_{1}} & \mathbf{0} \\
\mathbf{0} & -\boldsymbol{I}_{N_{2}}
\end{array}\right), \boldsymbol{a}=\left(\begin{array}{cc}
1 & 0 \\
0 & -1
\end{array}\right)
$$

By the forms of matrices $\boldsymbol{M}, \boldsymbol{r}$ and ${ }^{t} \boldsymbol{c}$ given in (2.3), one can easily recognize the following facts

$$
r^{t} c A=-A r^{t} c, K A=A K, M A=-A M,{ }^{t} c A=-a^{t} c, A r=r a, a^{2}=I_{2} .
$$

\section{The discrete nAKNS equation}

We suppose that $\boldsymbol{r}$ and $\boldsymbol{M}$ are functions of $(n, m)$ while ${ }^{t} \boldsymbol{c}$ and $\boldsymbol{K}$ are non-trivial constant matrices. The shifts of $\boldsymbol{r}$ are set as

$$
\begin{aligned}
& (p \boldsymbol{I}-\boldsymbol{A} \boldsymbol{K}) \widetilde{\boldsymbol{r}}=(p \boldsymbol{I}+\boldsymbol{A} \boldsymbol{K}) \boldsymbol{r}, \\
& \left(q \boldsymbol{I}-\boldsymbol{A} \boldsymbol{K}^{-1}\right) \widehat{\boldsymbol{r}}=\left(q \boldsymbol{I}+\boldsymbol{A} \boldsymbol{K}^{-1}\right) \boldsymbol{r},
\end{aligned}
$$

where $\boldsymbol{A}$ is defined by (2.19). In (3.1) by ${ }^{\sim}$ and ${ }^{\wedge}$ we respectively denote the shifts in $n$ and $m$ directions, i.e., $\widetilde{\boldsymbol{r}}(n, m)=\boldsymbol{r}(n+1, m), \widehat{\boldsymbol{r}}(n, m)=\boldsymbol{r}(n, m+1)$. For convenience, we call the gather of the Sylvester equation (2.2) and system (3.1): determining equation set.

\subsection{Shifts of $M$}

Proposition 3.1. For $\boldsymbol{M}, \boldsymbol{K}, \boldsymbol{r}$ and ${ }^{t} \boldsymbol{c}$ obeying the determining equation set (2.2) and (3.1), one has relations

$$
\begin{aligned}
& (p \boldsymbol{I}-\boldsymbol{A} \boldsymbol{K}) \widetilde{\boldsymbol{M}}=(p \boldsymbol{I}+\boldsymbol{A} \boldsymbol{K}) \boldsymbol{M} \\
& \left(q \boldsymbol{I}-\boldsymbol{A} \boldsymbol{K}^{-1}\right) \widehat{\boldsymbol{M}}=\left(q \boldsymbol{I}+\boldsymbol{A} \boldsymbol{K}^{-1}\right) \boldsymbol{M}
\end{aligned}
$$


and

$$
\begin{aligned}
& \widetilde{\boldsymbol{M}}(p \boldsymbol{I}+\boldsymbol{A} \boldsymbol{K})-(p \boldsymbol{I}+\boldsymbol{A} \boldsymbol{K}) \boldsymbol{M}=\widetilde{\boldsymbol{r}} \boldsymbol{a}^{t} \boldsymbol{c}, \\
& (p \boldsymbol{I}-\boldsymbol{A} \boldsymbol{K}) \widetilde{\boldsymbol{M}}-\boldsymbol{M}(p \boldsymbol{I}-\boldsymbol{A} \boldsymbol{K})=\boldsymbol{r} \boldsymbol{a}^{t} \boldsymbol{c}, \\
& -\widehat{\boldsymbol{M}}\left(q \boldsymbol{I}+\boldsymbol{A} \boldsymbol{K}^{-1}\right)-\left(q \boldsymbol{I}+\boldsymbol{A} \boldsymbol{K}^{-1}\right) \boldsymbol{M}=\boldsymbol{K}^{-1} \widehat{\boldsymbol{r}} \boldsymbol{a}^{t} \boldsymbol{c} \boldsymbol{K}^{-1}, \\
& \boldsymbol{M}\left(q \boldsymbol{I}-\boldsymbol{A} \boldsymbol{K}^{-1}\right)-\left(q \boldsymbol{I}-\boldsymbol{A} \boldsymbol{K}^{-1}\right) \widehat{\boldsymbol{M}}=\boldsymbol{K}^{-1} \boldsymbol{r} \boldsymbol{a}^{t} \boldsymbol{c} \boldsymbol{K}^{-1} .
\end{aligned}
$$

Proof. Taking ${ }^{\sim}$-shift of (2.2) and using (3.1a), we have

$$
\boldsymbol{K}(p \boldsymbol{I}-\boldsymbol{A} \boldsymbol{K}) \widetilde{\boldsymbol{M}}-(p \boldsymbol{I}-\boldsymbol{A} \boldsymbol{K}) \widetilde{\boldsymbol{M}} \boldsymbol{K}=(p \boldsymbol{I}+\boldsymbol{A} \boldsymbol{K}) \boldsymbol{r}^{t} \boldsymbol{c} .
$$

Replacing $\boldsymbol{r}^{t} \boldsymbol{c}$ in (3.4) by using of (2.2) leads to

$$
\boldsymbol{K}((p \boldsymbol{I}-\boldsymbol{A} \boldsymbol{K}) \widetilde{\boldsymbol{M}}-(p \boldsymbol{I}+\boldsymbol{A} \boldsymbol{K}) \boldsymbol{M})-((p \boldsymbol{I}-\boldsymbol{A} \boldsymbol{K}) \widetilde{\boldsymbol{M}}-(p \boldsymbol{I}+\boldsymbol{A} \boldsymbol{K}) \boldsymbol{M}) \boldsymbol{K}=0,
$$

which indicates (3.2a) in the light of Proposition 2.1. In a similar way, one can derive equation (3.2b).

Next we prove (3.3). To begin, we consider equation

$$
A K M-A M K=r a^{t} c .
$$

Taking $\sim$ shift of (3.6) we have

$$
\widetilde{r} \boldsymbol{a}^{t} \boldsymbol{c}=\boldsymbol{A} \boldsymbol{K} \widetilde{\boldsymbol{M}}-\boldsymbol{A} \widetilde{M} \boldsymbol{K},
$$

and replacing term $\boldsymbol{A} \boldsymbol{K} \widetilde{\boldsymbol{M}}$ by (3.2a) yields

$$
\begin{aligned}
\widetilde{\boldsymbol{r}} \boldsymbol{a}^{t} \boldsymbol{c} & =p \widetilde{\boldsymbol{M}}-(p \boldsymbol{I}+\boldsymbol{A} \boldsymbol{K}) \boldsymbol{M}-\boldsymbol{A} \widetilde{\boldsymbol{M}} \boldsymbol{K} \\
& =\widetilde{\boldsymbol{M}}(p \boldsymbol{I}+\boldsymbol{A} \boldsymbol{K})-(p \boldsymbol{I}+\boldsymbol{A} \boldsymbol{K}) \boldsymbol{M},
\end{aligned}
$$

where we have used equality (2.20). Besides, deleting term $\boldsymbol{A} \boldsymbol{K} \boldsymbol{M}$ from (3.6) and (3.2a) we get

$$
\begin{aligned}
\boldsymbol{r a}^{t} \boldsymbol{c} & =(p \boldsymbol{I}-\boldsymbol{A} \boldsymbol{K}) \widetilde{\boldsymbol{M}}-p \boldsymbol{M}-\boldsymbol{A} \boldsymbol{M} \boldsymbol{K} \\
& =(p \boldsymbol{I}-\boldsymbol{A} \boldsymbol{K}) \widetilde{\boldsymbol{M}}-\boldsymbol{M}(p \boldsymbol{I}-\boldsymbol{A} \boldsymbol{K}),
\end{aligned}
$$

where equality (2.20) has been utilized.

By similar analysis, one can prove (3.3c) and (3.3d).

\subsection{Shifts of $S^{(i, j)}$}

Proposition 3.2. For $\boldsymbol{M}, \boldsymbol{K}, \boldsymbol{r}$ and ${ }^{t} \boldsymbol{c}$ obeying the determining equation set (2.2) and (3.1), one has relations

$$
\begin{aligned}
& p \widetilde{\boldsymbol{S}}^{(i, j)}+\boldsymbol{a} \widetilde{\boldsymbol{S}}^{(i, j+1)}=p \boldsymbol{S}^{(i, j)}+\boldsymbol{S}^{(i+1, j)} \boldsymbol{a}-\boldsymbol{S}^{(0, j)} \boldsymbol{a} \widetilde{\boldsymbol{S}}^{(i, 0)}, \\
& p \boldsymbol{S}^{(i, j)}-\boldsymbol{a} \boldsymbol{S}^{(i, j+1)}=p \widetilde{\boldsymbol{S}}^{(i, j)}-\widetilde{\boldsymbol{S}}^{(i+1, j)} \boldsymbol{a}+\widetilde{\boldsymbol{S}}^{(0, j)} \boldsymbol{a} \boldsymbol{S}^{(i, 0)} \\
& q \widehat{\boldsymbol{S}}^{(i, j)}+\boldsymbol{a} \widehat{\boldsymbol{S}}^{(i, j-1)}=q \boldsymbol{S}^{(i, j)}+\boldsymbol{S}^{(i-1, j)} \boldsymbol{a}+\boldsymbol{S}^{(-1, j)} \boldsymbol{a} \widehat{\boldsymbol{S}}^{(i,-1)} \\
& q \boldsymbol{S}^{(i, j)}-\boldsymbol{a} \boldsymbol{S}^{(i, j-1)}=q \widehat{\boldsymbol{S}}^{(i, j)}-\widehat{\boldsymbol{S}}^{(i-1, j)} \boldsymbol{a}-\widehat{\boldsymbol{S}}^{(-1, j)} \boldsymbol{a} \boldsymbol{S}^{(i,-1)} .
\end{aligned}
$$


Proof. We firstly consider the shifts of auxiliary matrix function $\boldsymbol{u}^{(i)}$ defined by (2.7). Notice that (2.7) is tantamount to

$$
(\boldsymbol{I}+\boldsymbol{M}) \boldsymbol{u}^{(i)}=\boldsymbol{K}^{i} \boldsymbol{r}
$$

Taking ${ }^{\sim}$-shift of (3.10) together with (3.1a) leads to

$$
(p \boldsymbol{I}-\boldsymbol{A} \boldsymbol{K}) \widetilde{\boldsymbol{u}}^{(i)}+(p \boldsymbol{I}-\boldsymbol{A} \boldsymbol{K}) \widetilde{\boldsymbol{M}} \widetilde{\boldsymbol{u}}^{(i)}=\boldsymbol{K}^{i}(p \boldsymbol{I}+\boldsymbol{A} \boldsymbol{K}) \boldsymbol{r} .
$$

Replacing term $(p \boldsymbol{I}-\boldsymbol{A} \boldsymbol{K}) \widetilde{\boldsymbol{M}}$ with the help of (3.3b), we derive

$$
(\boldsymbol{I}+\boldsymbol{M})(p \boldsymbol{I}-\boldsymbol{A} \boldsymbol{K}) \widetilde{\boldsymbol{u}}^{(i)}=p \boldsymbol{K}^{i} \boldsymbol{r}+\boldsymbol{K}^{i+1} \boldsymbol{r} \boldsymbol{a}-\boldsymbol{r} \boldsymbol{a}^{t} \widetilde{\boldsymbol{u}}^{(i)},
$$

which implies

$$
(p \boldsymbol{I}-\boldsymbol{A} \boldsymbol{K}) \widetilde{\boldsymbol{u}}^{(i)}=p \boldsymbol{u}^{(i)}+\boldsymbol{u}^{(i+1)} \boldsymbol{a}-\boldsymbol{u}^{(0)} \boldsymbol{a} \widetilde{\boldsymbol{S}}^{(i, 0)}
$$

after the left-multiplication $(\boldsymbol{I}+\boldsymbol{M})^{-1}$.

Multiplying (3.10) from the left by matrix $p \boldsymbol{I}+\boldsymbol{A} \boldsymbol{K}$ and using (3.1a) and (3.3a) give rise to

$$
(\boldsymbol{I}+\widetilde{\boldsymbol{M}})(p \boldsymbol{I}+\boldsymbol{A} \boldsymbol{K}) \boldsymbol{u}^{(i)}=p \boldsymbol{K}^{i} \widetilde{\boldsymbol{r}}-\boldsymbol{K}^{i+1} \widetilde{\boldsymbol{r}} \boldsymbol{a}+\widetilde{\boldsymbol{r}} \boldsymbol{a}^{t} \boldsymbol{c} \boldsymbol{u}^{(i)},
$$

which is

$$
(p \boldsymbol{I}+\boldsymbol{A} \boldsymbol{K}) \boldsymbol{u}^{(i)}=p \widetilde{\boldsymbol{u}}^{(i)}-\widetilde{\boldsymbol{u}}^{(i+1)} \boldsymbol{a}+\widetilde{\boldsymbol{u}}^{(0)} \boldsymbol{a} \boldsymbol{S}^{(i, 0)}
$$

after the left-multiplication $(\boldsymbol{I}+\widetilde{\boldsymbol{M}})^{-1}$. Equations (3.13) and (3.15) constitute the shifts of $\boldsymbol{u}^{(i)}$ w.r.t. discrete variable $n$. With similar discussion, we can have the shifts of $\boldsymbol{u}^{(i)}$ w.r.t. discrete variable $m$

$$
\begin{aligned}
& \left(q \boldsymbol{I}-\boldsymbol{A} \boldsymbol{K}^{-1}\right) \widehat{\boldsymbol{u}}^{(i)}=q \boldsymbol{u}^{(i)}+\boldsymbol{u}^{(i-1)} \boldsymbol{a}+\boldsymbol{u}^{(-1)} \boldsymbol{a} \widehat{\boldsymbol{S}}^{(i,-1)}, \\
& \left(q \boldsymbol{I}+\boldsymbol{A} \boldsymbol{K}^{-1}\right) \boldsymbol{u}^{(i)}=q \widehat{\boldsymbol{u}}^{(i)}-\widehat{\boldsymbol{u}}^{(i-1)} \boldsymbol{a}-\widehat{\boldsymbol{u}}^{(-1)} \boldsymbol{a} \widetilde{\boldsymbol{S}}^{(i,-1)} .
\end{aligned}
$$

Left-multiplying (3.13), (3.15) and (3.16) by the matrix ${ }^{t} \boldsymbol{c} \boldsymbol{K}^{j}$, respectively and noting (2.20) together with the relation (2.8), one can derive the shifts (3.9) of matrix function $\boldsymbol{S}^{(i, j)}$ immediately.

For the sake of the resulting closed form equation, we introduce the following objects

$$
\begin{aligned}
& \boldsymbol{u}=\boldsymbol{S}^{(0,0)}=\left(\begin{array}{ll}
u_{1} & u_{2} \\
u_{3} & u_{4}
\end{array}\right), \\
& \boldsymbol{v}=\boldsymbol{I}_{2}-\boldsymbol{S}^{(-1,0)}, \boldsymbol{w}=\boldsymbol{S}^{(0,-1)}+\boldsymbol{I}_{2},
\end{aligned}
$$

where $u_{i},(i=1,2,3,4)$ are scalar functions. 


\subsection{The discrete $n A K N S$ equation}

To proceed, we take $i=j=0$ in (3.9c) and (3.9d) and have

$$
\boldsymbol{v} a \widehat{\boldsymbol{w}}=\widehat{\boldsymbol{v}} \boldsymbol{a} \boldsymbol{w}=\boldsymbol{a}-q(\widehat{\boldsymbol{u}}-\boldsymbol{u}) .
$$

In (3.9a) and (3.9b), the indices $i=-1, j=0$, respectively, $i=0, j=-1$ yield

$$
\begin{aligned}
p(\widetilde{\boldsymbol{v}}-\boldsymbol{v}) & =\boldsymbol{a} \widetilde{\boldsymbol{S}}^{(-1,1)}-\boldsymbol{u} \boldsymbol{a} \widetilde{\boldsymbol{v}}, \\
& =\boldsymbol{a} \boldsymbol{S}^{(-1,1)}-\widetilde{\boldsymbol{u}} \boldsymbol{a} \boldsymbol{v},
\end{aligned}
$$

and

$$
\begin{aligned}
p(\widetilde{\boldsymbol{w}}-\boldsymbol{w}) & =\boldsymbol{S}^{(1,-1)} \boldsymbol{a}-\boldsymbol{w a} \widetilde{\boldsymbol{u}}, \\
& =\boldsymbol{a} \boldsymbol{S}^{(-1,1)}-\widetilde{\boldsymbol{u}} \boldsymbol{a v} .
\end{aligned}
$$

Now noticing that (2.16) with $i=j=0 ; i=1, j=0$ and $i=0, j=1$, respectively, are

$$
\boldsymbol{v} \boldsymbol{w}=\boldsymbol{I}_{2}, \boldsymbol{S}^{(1,-1)}=\boldsymbol{w} \boldsymbol{u}, \boldsymbol{S}^{(-1,1)}=\boldsymbol{u v},
$$

then (3.19a) and (3.19b) turn out to be

$$
\begin{aligned}
p(\widetilde{\boldsymbol{v}}-\boldsymbol{v}) & =(\boldsymbol{a} \widetilde{\boldsymbol{u}}-\boldsymbol{u} \boldsymbol{a}) \widetilde{\boldsymbol{v}} \\
& =(\boldsymbol{a} \boldsymbol{u}-\widetilde{\boldsymbol{u}} \boldsymbol{a}) \boldsymbol{v}
\end{aligned}
$$

and

$$
\begin{aligned}
p(\widetilde{\boldsymbol{w}}-\boldsymbol{w}) & =\boldsymbol{w}(\boldsymbol{u} \boldsymbol{a}-\boldsymbol{a} \widetilde{\boldsymbol{u}}), \\
& =\widetilde{\boldsymbol{w}}(\widetilde{\boldsymbol{u}} \boldsymbol{a}-\boldsymbol{a} \boldsymbol{u}),
\end{aligned}
$$

or moreover

$$
\begin{aligned}
& p \boldsymbol{v} \widetilde{\boldsymbol{w}}=p \boldsymbol{I}_{2}+\boldsymbol{u} \boldsymbol{a}-\boldsymbol{a} \widetilde{\boldsymbol{u}}, \\
& p \widetilde{\boldsymbol{v}} \boldsymbol{w}=p \boldsymbol{I}_{2}+\boldsymbol{a} \boldsymbol{u}-\widetilde{\boldsymbol{u}} \boldsymbol{a} .
\end{aligned}
$$

Substituting (3.18) and (3.22) into relation

$$
(\boldsymbol{v} a \widehat{\boldsymbol{w}})^{\sim}(\widetilde{\boldsymbol{v}} \boldsymbol{w})^{\wedge}=(\widetilde{\boldsymbol{v}} \boldsymbol{w})(\boldsymbol{v} a \widehat{\boldsymbol{w}}),
$$

we get

$$
(\boldsymbol{a}-q(\widehat{\widetilde{\boldsymbol{u}}}-\widetilde{\boldsymbol{u}}))\left(p \boldsymbol{I}_{2}+\boldsymbol{a} \widehat{\boldsymbol{u}}-\widehat{\widetilde{\boldsymbol{u}}} \boldsymbol{a}\right)=\left(p \boldsymbol{I}_{2}+\boldsymbol{a} \boldsymbol{u}-\widetilde{\boldsymbol{u}} \boldsymbol{a}\right)(\boldsymbol{a}-q(\widehat{\boldsymbol{u}}-\boldsymbol{u})) .
$$

Besides, from (3.18) and (3.22), one knows that

$$
\begin{aligned}
& (\boldsymbol{a}-q(\widehat{\boldsymbol{u}}-\boldsymbol{u}))^{2}=\boldsymbol{I}_{2}, \\
& \left(p \boldsymbol{I}_{2}+\boldsymbol{u} \boldsymbol{a}-\boldsymbol{a} \widetilde{\boldsymbol{u}}\right)\left(p \boldsymbol{I}_{2}+\boldsymbol{a} \boldsymbol{u}-\widetilde{\boldsymbol{u}} \boldsymbol{a}\right)=p^{2} \boldsymbol{I}_{2} .
\end{aligned}
$$


Taking (3.17a) into (3.23) and using (3.24), one can easily get a system composed by $u_{2}$ and $u_{3}$ :

$$
\begin{aligned}
& q\left(\widehat{u}_{2}-u_{2}\right)\left[p^{2}-\left(u_{2}+\widetilde{u}_{2}\right)\left(u_{3}+\widetilde{u}_{3}\right)\right]^{\frac{1}{2}}+\left(u_{2}+\widetilde{u}_{2}\right)\left[1-q^{2}\left(\widehat{u}_{2}-u_{2}\right)\left(\widehat{u}_{3}-u_{3}\right)\right]^{\frac{1}{2}} \\
& +\left(\widehat{\widetilde{u}}_{2}+\widehat{\widetilde{u}}_{2}\right)\left[1-q^{2}\left(\widehat{\widetilde{u}}_{2}-\widetilde{u}_{2}\right)\left(\widehat{\widetilde{u}}_{3}-\widetilde{u}_{3}\right)\right]^{\frac{1}{2}}+q\left(\widetilde{\widetilde{u}}_{2}-\widehat{\widetilde{u}}_{2}\right)\left[p^{2}-\left(\widehat{\widetilde{u}}_{2}+\widehat{\widetilde{u}}_{2}\right)\left(\widehat{\widetilde{u}}_{3}+\widehat{\widetilde{u}}_{3}\right)\right]^{\frac{1}{2}}=0, \\
& q\left(\widehat{u}_{3}-u_{3}\right)\left[p^{2}-\left(u_{2}+\widetilde{u}_{2}\right)\left(u_{3}+\widetilde{u}_{3}\right)\right]^{\frac{1}{2}}+\left(u_{3}+\widetilde{u}_{3}\right)\left[1-q^{2}\left(\widehat{u}_{2}-u_{2}\right)\left(\widehat{u}_{3}-u_{3}\right)\right]^{\frac{1}{2}} \\
& +\left(\widehat{u}_{3}+\widehat{\widetilde{u}}_{3}\right)\left[1-q^{2}\left(\widehat{\widetilde{u}}_{2}-\widetilde{u}_{2}\right)\left(\widehat{\widetilde{u}}_{3}-\widetilde{u}_{3}\right)\right]^{\frac{1}{2}}+q\left(\widetilde{u}_{3}-\widehat{\widetilde{u}}_{3}\right)\left[p^{2}-\left(\widehat{u}_{2}+\widehat{\widetilde{u}}_{2}\right)\left(\widehat{u}_{3}+\widehat{\widetilde{u}}_{3}\right)\right]^{\frac{1}{2}}=0,
\end{aligned}
$$

which we refer to the discrete nAKNS equation, whose solutions $u_{2}$ and $u_{3}$ are given by

$$
\begin{aligned}
& u_{2}={ }^{t} \boldsymbol{c}_{2}\left(\boldsymbol{I}_{N_{2}}-\boldsymbol{M}_{2} \boldsymbol{M}_{1}\right)^{-1} \boldsymbol{r}_{2}, \\
& u_{3}={ }^{t} \boldsymbol{c}_{1}\left(\boldsymbol{I}_{N_{1}}-\boldsymbol{M}_{1} \boldsymbol{M}_{2}\right)^{-1} \boldsymbol{r}_{1} .
\end{aligned}
$$

In the following our task is to solve the determining equation set (2.2) and (3.1) so that we can give explicit solutions of the above lattice equation.

\section{Exact solutions}

We firstly introduce some notations, where usually the subscripts ${ }_{D}$ and ${ }_{J}$ correspond to the cases of $\Gamma$ being diagonal and being of Jordan block, respectively.

\subsection{Some special matrices}

- Diagonal matrix:

$$
\Gamma_{D}^{[N}\left(\left\{k_{i}\right\}_{1}^{N}\right)=\operatorname{Diag}\left(k_{1}, k_{2}, \ldots, k_{N}\right)
$$

- Jordan block matrix:

$$
\Gamma_{J}^{[N}(a)=\left(\begin{array}{cccccc}
a & 0 & 0 & \cdots & 0 & 0 \\
1 & a & 0 & \cdots & 0 & 0 \\
0 & 1 & a & \cdots & 0 & 0 \\
\vdots & \vdots & \vdots & \vdots & \vdots & \vdots \\
0 & 0 & 0 & \cdots & 1 & a
\end{array}\right)_{N \times N}
$$

- Lower triangular Toeplitz matrix: ${ }^{\mathrm{a}}$

$$
\boldsymbol{T}^{[N \mid}\left(\left\{a_{j}\right\}_{1}^{N}\right)=\left(\begin{array}{cccccc}
a_{1} & 0 & 0 & \cdots & 0 & 0 \\
a_{2} & a_{1} & 0 & \cdots & 0 & 0 \\
a_{3} & a_{2} & a_{1} & \cdots & 0 & 0 \\
\vdots & \vdots & \cdots & \vdots & \vdots & \vdots \\
a_{N} & a_{N-1} & a_{N-2} & \cdots & a_{2} & a_{1}
\end{array}\right)_{N \times N},
$$

\footnotetext{
${ }^{\mathrm{a}}$ More properties of this kind of matrices can be found in [29].
} 
- Skew triangular Hankel matrix:

$$
\boldsymbol{H}^{[N]}\left(\left\{b_{j}\right\}_{1}^{N}\right)=\left(\begin{array}{ccccc}
b_{1} & \cdots & b_{N-2} & b_{N-1} & b_{N} \\
b_{2} & \cdots & b_{N-1} & b_{N} & 0 \\
b_{3} & \cdots & b_{N} & 0 & 0 \\
\vdots & \vdots & \vdots & \vdots & \vdots \\
b_{N} & \cdots & 0 & 0 & 0
\end{array}\right)_{N \times N} .
$$

\subsection{Solutions to the Sylvester equation (2.2)}

By virtue of the invariance of $\boldsymbol{S}^{(i, j)}$ under similar transformation (2.17), we just need to solve the following canonical equation

$$
\boldsymbol{\Omega M}-\mathbf{M} \Omega=\boldsymbol{r}^{t} \boldsymbol{c}
$$

where $\boldsymbol{\Omega}=\operatorname{Diag}(\boldsymbol{\Gamma}, \boldsymbol{\Lambda})$ is the canonical form of matrix $\boldsymbol{K}=\operatorname{Diag}\left(\boldsymbol{K}_{1}, \boldsymbol{K}_{2}\right)$. Corresponding to condition $\mathscr{E}\left(\boldsymbol{K}_{1}\right) \cap \mathscr{E}\left(\boldsymbol{K}_{2}\right)=\varnothing$, hereafter we assume $\mathscr{E}(\boldsymbol{\Gamma}) \cap \mathscr{E}(\boldsymbol{\Lambda})=\varnothing$. Because both $\boldsymbol{\Gamma}$ and $\boldsymbol{\Lambda}$ are of form canonical structures, it is possible to give a complete classification for the solutions. Besides those matrix notations we used in the previous section, let us list other needed ones below.

$$
\begin{aligned}
& 2 N \times 2 N \text { matrix : } \boldsymbol{G}_{D D}^{\left[N_{1}: N_{2}\right]}\left(\left\{k_{i}\right\}_{1}^{N_{1}} ;\left\{l_{j}\right\}_{1}^{N_{2}}\right) \\
& =\left(\begin{array}{cc}
\mathbf{0} & \boldsymbol{G}_{D D ; 12}^{\left[N_{1} ; N_{2}\right]}\left(\left\{k_{i}\right\}_{1}^{N_{1}} ;\left\{l_{j}\right\}_{1}^{N_{2}}\right) \\
-\boldsymbol{G}_{D D ; 2}^{\left[N_{1} ; N_{2}\right]^{T}}\left(\left\{k_{i}\right\}_{1}^{N_{1}} ;\left\{l_{j}\right\}_{1}^{N_{2}}\right) & \mathbf{0}
\end{array}\right), \\
& \boldsymbol{G}_{D D ; 2}^{\left[N_{1} ; N_{2}\right]}\left(\left\{k_{i}\right\}_{1}^{N_{1}} ;\left\{l_{j}\right\}_{1}^{N_{2}}\right)=\left(\frac{1}{k_{i}-l_{j}}\right)_{N_{1} \times N_{2}}, \\
& 2 N \times 2 N \text { matrix : } \boldsymbol{G}_{D J}^{\left[N_{1} ; N_{2}\right]}\left(\left\{k_{i}\right\}_{1}^{N_{1}} ; b\right)=\left(\begin{array}{cc}
\mathbf{0} & \boldsymbol{G}_{D j ; 12}^{\left[N_{1}, N_{2}\right]}\left(\left\{k_{i}\right\}_{1}^{N_{1}} ; b\right) \\
-\boldsymbol{G}_{D J ; j 2}^{\left[N_{1} ; N_{2}\right]^{T}}\left(\left\{k_{i}\right\}_{1}^{N_{1}} ; b\right) & \mathbf{0}
\end{array}\right), \\
& \boldsymbol{G}_{D j ; 12}^{\left[N_{1} ; N_{2}\right]}\left(\left\{k_{j}\right\}_{1}^{N_{1}} ; b\right)=\left(g_{i, j}\right)_{N_{1} \times N_{2}}, \quad g_{i, j}=\left(\frac{1}{k_{i}-b}\right)^{j}, \\
& 2 N \times 2 N \text { matrix : } \boldsymbol{G}_{J D}^{\left[N_{1}: N_{2}\right]}\left(a ;\left\{l_{j}\right\}_{1}^{N_{2}}\right)=\left(\begin{array}{cc}
\mathbf{0} & \boldsymbol{G}_{J D ; 12}^{\left[N_{1}: N_{2}\right]}\left(a ;\left\{l_{j}\right\}_{1}^{N_{2}}\right) \\
-\boldsymbol{G}_{J D ; 12}^{\left[N_{1}: N_{2}\right]^{T}}\left(a ;\left\{l_{j}\right\}_{1}^{N_{2}}\right) & \mathbf{0}
\end{array}\right) \text {, } \\
& \boldsymbol{G}_{J D ; 12}^{\left[N_{1}, N_{2}\right]}\left(a ;\left\{l_{j}\right\}_{1}^{N_{2}}\right)=\left(g_{i, j}\right)_{N_{1} \times N_{2}}, \quad g_{i, j}=-\left(\frac{-1}{a-l_{j}}\right)^{i}, \\
& 2 N \times 2 N \text { matrix : } \boldsymbol{G}_{J J}^{\left[N_{1}: N_{2}\right]}(a ; b)=\left(\begin{array}{cc}
\mathbf{0}^{T} & \boldsymbol{G}_{J j ; 12}^{\left[N_{1}: N_{2}\right]}(a ; b) \\
-\boldsymbol{G}_{J j ; 12}^{\left[N_{1}: N_{2}\right]^{T}}(a ; b) & \mathbf{0}
\end{array}\right), \\
& \boldsymbol{G}_{J j ; 1 / 2}^{\left[N_{1}: N_{2}\right]}(a ; b)=\left(g_{i, j}\right)_{N_{1} \times N_{2}}, \quad g_{i, j}=\mathrm{C}_{i+j-2}^{i-1} \frac{(-1)^{i+1}}{(a-b)^{i+j-1}},
\end{aligned}
$$

where

$$
\mathrm{C}_{j}^{i}=\frac{j !}{i !(j-i) !}, \quad(j \geq i) .
$$

The procedure for solving the Sylvester equation (2.2) can be found in [31]. The key point of the solving procedure is to factorize $\boldsymbol{M}$ into a triplet, i.e. $\boldsymbol{M}=\boldsymbol{F} \boldsymbol{G H}$. Here we skip the detailed procedure and directly write out solutions. 
Proposition 4.1. (1). When

$$
\boldsymbol{\Gamma}=\boldsymbol{\Gamma}_{D}^{\left[N_{1}\right]}\left(\left\{k_{i}\right\}_{1}^{N_{1}}\right), \boldsymbol{\Lambda}=\boldsymbol{\Gamma}_{D}^{\left[N_{2}\right]}\left(\left\{l_{j}\right\}_{1}^{N_{2}}\right),
$$

we have

$$
\boldsymbol{M}=\boldsymbol{F} \boldsymbol{G H},
$$

where

$$
\begin{aligned}
& \boldsymbol{F}=\operatorname{Diag}\left(\boldsymbol{\Gamma}_{D}^{\left[N_{1}\right]}\left(\left\{r_{1, i}\right\}_{1}^{N_{1}}\right), \boldsymbol{\Gamma}_{D}^{\left[N_{2}\right]}\left(\left\{r_{2, j}\right\}_{1}^{N_{2}}\right)\right), \\
& \boldsymbol{G}=\boldsymbol{G}_{D D}^{\left[N_{1} N_{2}\right]}\left(\left\{k_{i}\right\}_{1}^{N_{1}} ;\left\{l_{j}\right\}_{1}^{N_{2}}\right), \\
& \boldsymbol{H}=\operatorname{Diag}\left(\boldsymbol{\Gamma}_{D}^{\left(N_{1}\right]}\left(\left\{c_{1, i}\right\}_{1}^{N_{1}}\right), \boldsymbol{\Gamma}_{D}^{\left[N_{2} l\right.}\left(\left\{c_{2, j}\right\}_{1}^{N_{2}}\right)\right) .
\end{aligned}
$$

(2). When

$$
\boldsymbol{\Gamma}=\boldsymbol{\Gamma}_{D}^{\left[N_{1}\right]}\left(\left\{k_{i}\right\}_{1}^{N_{1}}\right), \boldsymbol{\Lambda}=\boldsymbol{\Gamma}_{J}^{\left[N_{2}\right]}\left(l_{1}\right),
$$

we have

$$
\boldsymbol{M}=\boldsymbol{F} \boldsymbol{G H},
$$

where

$$
\begin{aligned}
& \boldsymbol{F}=\operatorname{Diag}\left(\boldsymbol{\Gamma}_{D}^{\left[N_{1}\right]}\left(\left\{r_{1, i}\right\}_{1}^{N_{1}}\right), \boldsymbol{T}^{\left[N_{2}\right]}\left(\left\{r_{2, j}\right\}_{1}^{N_{2}}\right)\right), \\
& \boldsymbol{G}=\boldsymbol{G}_{D J}^{\left[N_{1} N_{2}\right]}\left(\left\{k_{i}\right\}_{1}^{N_{1}} ; l_{1}\right), \\
& \boldsymbol{H}=\operatorname{Diag}\left(\boldsymbol{\Gamma}_{D}^{\left.N_{1}\right]}\left(\left\{c_{1, i}\right\}_{1}^{N_{1}}\right), \boldsymbol{H}^{\left[N_{2}\right]}\left(\left\{c_{2, j}\right\}_{1}^{N_{2}}\right)\right) .
\end{aligned}
$$

Likewise, we can also obtain the solution for (4.5) when $\boldsymbol{\Gamma}=\boldsymbol{\Gamma}_{J}^{\left[N_{1}\right]}\left(k_{1}\right), \boldsymbol{\Lambda}=\boldsymbol{\Gamma}_{D}^{\left[N_{2}\right]}\left(\left\{l_{j}\right\}_{1}^{N_{2}}\right)$.

(3). When

$$
\Gamma=\Gamma_{J}^{\left[N_{1}\right]}\left(k_{1}\right), \boldsymbol{\Lambda}=\Gamma_{J}^{\left[N_{2}\right]}\left(l_{1}\right),
$$

we have

$$
\boldsymbol{M}=\boldsymbol{F} \boldsymbol{G H},
$$

where

$$
\begin{aligned}
& \boldsymbol{F}=\operatorname{Diag}\left(\boldsymbol{T}^{\left[N_{1} l\right.}\left(\left\{r_{1, j}\right\}_{1}^{N_{1}}\right), \boldsymbol{T}^{\left[N_{2}\right]}\left(\left\{r_{2, j}\right\}_{1}^{N_{2}}\right)\right), \\
& \boldsymbol{G}=\boldsymbol{G}_{J J}^{\left[N_{1}: N_{2}\right]}\left(k_{1} ; l_{1}\right), \\
& \boldsymbol{H}=\operatorname{Diag}\left(\boldsymbol{H}^{\left[N_{1}\right]}\left(\left\{c_{1, j}\right\}_{1}^{N_{1}}\right), \boldsymbol{H}^{\left[N_{2}\right]}\left(\left\{c_{2, j}\right\}_{1}^{N_{2}}\right)\right) .
\end{aligned}
$$

(4). When

$$
\begin{aligned}
& \boldsymbol{\Gamma}=\operatorname{Diag}\left(\boldsymbol{\Gamma}_{D}^{\left[N_{11}\right]}\left(\left\{k_{i}\right\}_{1}^{N_{11}}\right), \boldsymbol{\Gamma}_{J}^{\left[N_{12}\right]}\left(k_{N_{11}+1}\right), \boldsymbol{\Gamma}_{J}^{\left[N_{13}\right]}\left(k_{N_{11}+2}\right), \cdots, \boldsymbol{\Gamma}_{J}^{\left[N_{15} l\right.}\left(k_{N_{11}+(s-1)}\right)\right), \\
& \boldsymbol{\Lambda}=\operatorname{Diag}\left(\boldsymbol{\Gamma}_{D}^{\left[N_{21}\right]}\left(\left\{l_{j}\right\}_{1}^{N_{21}}\right), \boldsymbol{\Gamma}_{J}^{\left[N_{22}\right]}\left(l_{N_{21}+1}\right), \boldsymbol{\Lambda}_{J}^{\left[N_{23}\right]}\left(l_{N_{21}+2}\right), \cdots, \boldsymbol{\Gamma}_{J}^{\left[N_{25}\right]}\left(l_{N_{21}+(s-1)}\right)\right),
\end{aligned}
$$

where $\sum_{j=1}^{s} N_{i j}=N_{i}(i=1,2)$, we have

$$
\boldsymbol{M}=\boldsymbol{F} \boldsymbol{G H},
$$


where

$$
\boldsymbol{F}=\operatorname{Diag}\left(\boldsymbol{F}_{1}, \boldsymbol{F}_{2}\right), \quad \boldsymbol{H}=\operatorname{Diag}\left(\boldsymbol{H}_{1}, \boldsymbol{H}_{2}\right),
$$

with

$$
\begin{aligned}
& \boldsymbol{F}_{1}=\operatorname{Diag}\left(\boldsymbol{\Gamma}_{D}^{\left[N_{11}\right]}\left(\left\{r_{1, j}\right\}_{1}^{N_{11}}\right), \boldsymbol{T}^{\left[N_{12}\right]}\left(\left\{r_{1, j}\right\}_{N_{11}+1}^{N_{11}+N_{12}}\right), \ldots, \boldsymbol{T}^{\left[N_{1 s}\right]}\left(\left\{r_{1, j}\right\}_{\sum_{j=1}^{s-1} N_{1 j}+1}^{N_{1}}\right)\right), \\
& \boldsymbol{F}_{2}=\operatorname{Diag}\left(\boldsymbol{\Gamma}_{D}^{\left[N_{21}\right]}\left(\left\{r_{2, j}\right\}_{1}^{N_{21}}\right), \boldsymbol{T}^{\left[N_{22}\right]}\left(\left\{r_{2, j}\right\}_{N_{21}+1}^{N_{21}+N_{22}}\right), \ldots, \boldsymbol{T}^{\left[N_{2 s}\right]}\left(\left\{r_{2, j}\right\}_{\sum_{j=1}^{s-1} N_{2 j}+1}^{N_{2}}\right)\right), \\
& \boldsymbol{H}_{1}=\operatorname{Diag}\left(\boldsymbol{\Gamma}_{D}^{\left[N_{11}\right]}\left(\left\{c_{1, j}\right\}_{1}^{N_{11}}\right), \boldsymbol{H}^{\left[N_{12}\right]}\left(\left\{c_{1, j}\right\}_{N_{11}+1}^{N_{11}+N_{12}}\right), \ldots, \boldsymbol{H}^{\left[N_{1 s}\right]}\left(\left\{c_{1, j}\right\}_{\sum_{j=1}^{s-1} N_{1 j}+1}^{N_{1}}\right)\right), \\
& \boldsymbol{H}_{2}=\operatorname{Diag}\left(\boldsymbol{\Gamma}_{D}^{\left[N_{21}\right]}\left(\left\{c_{2, j}\right\}_{1}^{N_{21}}\right), \boldsymbol{H}^{\left[N_{22}\right]}\left(\left\{c_{2, j}\right\}_{N_{21}+1}^{N_{21}+N_{22}}\right), \ldots, \boldsymbol{H}^{\left[N_{2 s}\right]}\left(\left\{c_{2, j}\right\}_{\sum_{j=1}^{s-1} N_{2 j}+1}^{N_{2}}\right)\right),
\end{aligned}
$$

and $\boldsymbol{G}$ is of form

$$
\boldsymbol{G}=\left(\begin{array}{cc}
\mathbf{0} & \boldsymbol{G}_{1} \\
-\boldsymbol{G}_{1}^{T} & \mathbf{0}
\end{array}\right)
$$

where

$$
\boldsymbol{G}_{1}=\left(\boldsymbol{G}_{1}^{(i, j)}\right)_{s \times s}
$$

with

$$
\begin{array}{ll}
\boldsymbol{G}_{1}^{(1,1)}=\boldsymbol{G}_{D D}^{\left[N_{11}: N_{21}\right]}\left(\left\{k_{i}\right\}_{1}^{N_{11}} ;\left\{l_{j}\right\}_{1}^{N_{21}}\right), & \\
\boldsymbol{G}_{1}^{(1, j)}=\boldsymbol{G}_{D J}^{\left.N_{11}: N_{2 j}\right]}\left(\left\{k_{i}\right\}_{1}^{N_{11}} ; l_{N_{21}+j-1}\right), & (1<j \leq s), \\
\boldsymbol{G}_{1}^{(i, 1)}=\boldsymbol{G}_{J D}^{\left[N_{1 i}: N_{21}\right]}\left(k_{N_{11}+i-1} ;\left\{l_{j}\right\}_{1}^{N_{21}}\right), & (1<i \leq s), \\
\boldsymbol{G}_{1}^{(i, j)}=\boldsymbol{G}_{J J}^{\left[N_{1 j}: N_{2 j}\right]}\left(k_{N_{11}+i-1} ; l_{N_{21}+j-1}\right), & (1<i, j \leq s) .
\end{array}
$$

\subsection{Solutions to the shift equations (3.1)}

Under similar transformation (2.17), shift equation (3.1) is covariant (cf. [31]), which implies one just needs to solve

$$
\begin{aligned}
& (p \boldsymbol{I}-\boldsymbol{A} \boldsymbol{\Omega}) \widetilde{\boldsymbol{r}}=(p \boldsymbol{I}+\boldsymbol{A} \boldsymbol{\Omega}) \boldsymbol{r}, \\
& \left(q \boldsymbol{I}-\boldsymbol{A} \boldsymbol{\Omega}^{-1}\right) \widehat{\boldsymbol{r}}=\left(q \boldsymbol{I}+\boldsymbol{A} \boldsymbol{\Omega}^{-1}\right) \boldsymbol{r},
\end{aligned}
$$

where $\Omega$ is defined by (4.5).

We already have solution $\boldsymbol{M}$ for the canonical Sylvester equation (4.5), where $\boldsymbol{M}$ is factorized as

$$
\boldsymbol{M}=\boldsymbol{F} \boldsymbol{G H},
$$

in which $\boldsymbol{F}$ is related to $\boldsymbol{r}, \boldsymbol{H}$ is related to ${ }^{t} \boldsymbol{c}$ and $\boldsymbol{G}$ is related to $\boldsymbol{\Gamma}$ and $\boldsymbol{\Lambda}$. Once we get the solutions for $\boldsymbol{r}$ that satisfies the shift relations (6.2), we can have the explicit form for

$$
\boldsymbol{S}^{(i, j)}={ }^{t} \boldsymbol{c} \boldsymbol{\Omega}^{j}(\boldsymbol{I}+\boldsymbol{M})^{-1} \boldsymbol{\Omega}^{i} \boldsymbol{r}
$$

and then solutions $u_{2}$ and $u_{3}$ of the discrete nAKNS equation (3.25). 
We suppose

$$
\begin{aligned}
\rho_{i} & =\left(\frac{p+k_{i}}{p-k_{i}}\right)^{n}\left(\frac{q+k_{i}^{-1}}{q-k_{i}^{-1}}\right)^{m} \rho_{i}^{0}, \text { with constants } \rho_{i}^{0}, \\
\sigma_{j} & =\left(\frac{p-l_{j}}{p+l_{j}}\right)^{n}\left(\frac{q-l_{j}^{-1}}{q+l_{j}^{-1}}\right)^{m} \sigma_{j}^{0}, \text { with constants } \sigma_{j}^{0},
\end{aligned}
$$

which are viewed as discrete plane wave factors. Then we have the following results.

(1). When $\boldsymbol{\Gamma}$ and $\boldsymbol{\Lambda}$ are given by (4.7), i.e.

$$
\boldsymbol{\Gamma}=\Gamma_{D}^{\left[N_{1}\right]}\left(\left\{k_{i}\right\}_{1}^{N_{1}}\right), \quad \boldsymbol{\Lambda}=\boldsymbol{\Gamma}_{D}^{\left[N_{2}\right]}\left(\left\{l_{j}\right\}_{1}^{N_{2}}\right),
$$

we have

$$
\begin{aligned}
& \boldsymbol{r}=\left(\begin{array}{cc}
\boldsymbol{r}_{1, D}^{\left[N_{1}\right]}\left(\left\{k_{i}\right\}_{1}^{N_{1}}\right) & \mathbf{0} \\
\mathbf{0} & \boldsymbol{r}_{2, D}^{\left[N_{2}\right]}\left(\left\{l_{j}\right\}_{1}^{N_{2}}\right)
\end{array}\right)=\left(\begin{array}{cc}
r_{1,1}, r_{1,2}, \cdots, r_{1, N_{1}} & 0,0, \cdots, 0 \\
0,0, \cdots, 0 & r_{2,1}, r_{2,2}, \cdots, r_{2, N_{2}}
\end{array}\right)^{T}, \\
& r_{1, i}=\rho_{i}, r_{2, j}=\sigma_{j}, \quad i=1,2, \ldots, N_{1} ; j=1,2, \ldots, N_{2} .
\end{aligned}
$$

(2). When $\Gamma$ and $\Lambda$ are given by (4.9), i.e.

$$
\boldsymbol{\Gamma}=\boldsymbol{\Gamma}_{D}^{\left[N_{1}\right]}\left(\left\{k_{i}\right\}_{1}^{N_{1}}\right), \quad \boldsymbol{\Lambda}=\boldsymbol{\Gamma}_{J}^{\left[N_{2}\right]}\left(l_{1}\right),
$$

we have

$$
\begin{aligned}
& \boldsymbol{r}=\left(\begin{array}{cc}
\boldsymbol{r}_{1, D}^{\left[N_{1}\right]}\left(\left\{k_{i}\right\}_{1}^{N_{1}}\right) & \mathbf{0} \\
\mathbf{0} & \boldsymbol{r}_{2, j}^{\left[N_{2}\right]}\left(l_{1}\right)
\end{array}\right)=\left(\begin{array}{cc}
r_{1,1}, r_{1,2}, \cdots, r_{1, N_{1}} & 0,0, \cdots, 0 \\
0,0, \cdots, 0 & r_{2,1}, r_{2,2}, \cdots, r_{2, N_{2}}
\end{array}\right)^{T}, \\
& r_{1, i}=\rho_{i}, r_{2, j}=\frac{\partial_{l_{1}}^{j-1} \sigma_{1}}{(j-1) !}, i=1,2, \ldots, N_{1} ; j=1,2, \ldots, N_{2} .
\end{aligned}
$$

(3). When $\Gamma$ and $\Lambda$ are given by (4.11), i.e.

$$
\Gamma=\Gamma_{J}^{\left[N_{1}\right]}\left(k_{1}\right), \boldsymbol{\Lambda}=\boldsymbol{\Gamma}_{J}^{\left[N_{2}\right]}\left(l_{1}\right),
$$

we have

$$
\begin{aligned}
& \boldsymbol{r}=\left(\begin{array}{cc}
\boldsymbol{r}_{1, j}^{\left[N_{1}\right]}\left(k_{1}\right) & \mathbf{0} \\
\mathbf{0} & \boldsymbol{r}_{2, j}^{\left[N_{2}\right]}\left(l_{1}\right)
\end{array}\right)=\left(\begin{array}{cc}
r_{1,1}, r_{1,2}, \cdots, r_{1, N_{1}} & 0,0, \cdots, 0 \\
0,0, \cdots, 0 & r_{2,1}, r_{2,2}, \cdots, r_{2, N_{2}}
\end{array}\right)^{T}, \\
& r_{1, i}=\frac{\partial_{k_{1}}^{i-1} \rho_{1}}{(i-1) !}, r_{2, j}=\frac{\partial_{l_{1}}^{j-1} \sigma_{1}}{(j-1) !}, i=1,2, \ldots, N_{1} ; j=1,2, \ldots, N_{2} .
\end{aligned}
$$

(4). When $\Gamma$ and $\Lambda$ are given by (4.11), i.e.

$$
\begin{aligned}
& \boldsymbol{\Gamma}=\operatorname{Diag}\left(\boldsymbol{\Gamma}_{D}^{\left[N_{11}\right]}\left(\left\{k_{i}\right\}_{1}^{N_{11}}\right), \boldsymbol{\Gamma}_{J}^{\left[N_{12}\right]}\left(k_{N_{11}+1}\right), \boldsymbol{\Gamma}_{J}^{\left[N_{13}\right]}\left(k_{N_{11}+2}\right), \cdots, \boldsymbol{\Gamma}_{J}^{\left[N_{1 s}\right]}\left(k_{N_{11}+(s-1)}\right)\right), \\
& \boldsymbol{\Lambda}=\operatorname{Diag}\left(\boldsymbol{\Gamma}_{D}^{\left[N_{21}\right]}\left(\left\{l_{j}\right\}_{1}^{N_{21}}\right), \boldsymbol{\Gamma}_{J}^{\left[N_{22}\right]}\left(l_{N_{21}+1}\right), \boldsymbol{\Gamma}_{J}^{\left[N_{23}\right]}\left(l_{N_{21}+2}\right), \cdots, \boldsymbol{\Gamma}_{J}^{\left[N_{2 s}\right]}\left(l_{N_{21}+(s-1)}\right)\right),
\end{aligned}
$$


where $\sum_{j=1}^{s} N_{i j}=N_{i}(i=1,2)$, we have

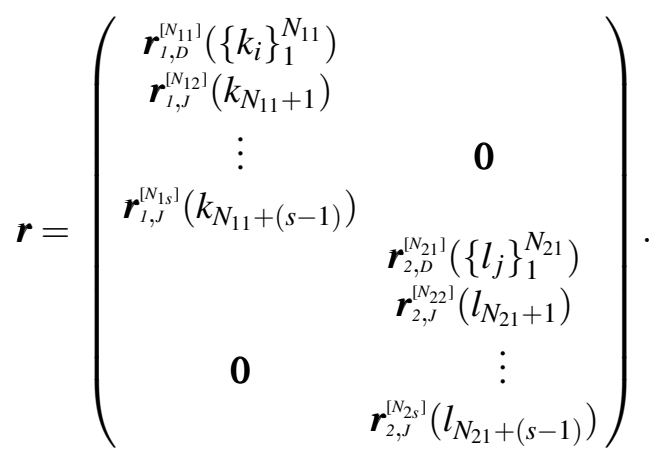

In summary, we obtain some types of exact solutions for the discrete nAKNS equation (3.25) by solving determining equation set (2.2) and (3.1). In Case 1 one can get the usual multi-soliton solutions. The solution in Case 3 gives rise to the Jordan block solutions or multiple-pole solutions, which can be viewed as limit solutions (See [27,29]). The solutions given by Case 2 and Case 4 are mixed solutions. The solution in Case 2 is the simplest one and the solution in Case 4 is the most general one, both of which in principle have properties of soliton and multiple-pole solution.

In next section, we consider continuum limit for the discrete nAKNS equation (3.25). For convenience, we turn to discuss continuum limit for the system (3.23) and (3.24).

\section{Continuum limit}

In Sec.3 we have given the discrete nAKNS equation (3.25). To obtain the corresponding differential-difference equation we consider the limit

$$
m \rightarrow \infty, q \rightarrow \infty, t=-\frac{m}{q} \sim O(1),
$$

in which $t$ can be identified as the continuous time variable. Then for the discrete plane-wave factor

$$
\rho=\left(\frac{p+\kappa}{p-\kappa}\right)^{n}\left(\frac{q+\kappa^{-1}}{q-\kappa^{-1}}\right)^{m} \rho^{0},
$$

we find

$$
\rho \rightarrow\left(\frac{p+\kappa}{p-\kappa}\right)^{n} e^{-2 \frac{t}{\kappa}} \rho^{0}
$$

and (3.23) together with (3.24) give rise to (in terms of $q^{0}$ and $\boldsymbol{u}=\boldsymbol{u}(n, t)$ )

$$
\begin{aligned}
& \left(\boldsymbol{a}+\widetilde{\boldsymbol{u}}_{t}\right)\left(p \boldsymbol{I}_{2}+\boldsymbol{a} \boldsymbol{u}-\widetilde{\boldsymbol{u}} \boldsymbol{a}\right)=\left(p \boldsymbol{I}_{2}+\boldsymbol{a} \boldsymbol{u}-\widetilde{\boldsymbol{u}} \boldsymbol{a}\right)\left(\boldsymbol{a}+\boldsymbol{u}_{t}\right), \\
& \left(\boldsymbol{a}+\boldsymbol{u}_{t}\right)^{2}=\boldsymbol{I}_{2}, \\
& \left(p \boldsymbol{I}_{2}+\boldsymbol{u} \boldsymbol{a}-\boldsymbol{a} \widetilde{\boldsymbol{u}}\right)\left(p \boldsymbol{I}_{2}+\boldsymbol{a} \boldsymbol{u}-\widetilde{\boldsymbol{u}} \boldsymbol{a}\right)=\boldsymbol{I}_{2} .
\end{aligned}
$$

The substitution of (3.17a) into (5.2) leads to the semi-discrete nAKNS equation

$$
\begin{aligned}
& \left(u_{2, t}-\widetilde{u}_{2, t}\right)\left[p^{2}-\left(u_{2}+\widetilde{u}_{2}\right)\left(u_{3}+\widetilde{u}_{3}\right)\right]^{\frac{1}{2}}+\left(u_{2}+\widetilde{u}_{2}\right)\left[\left(1-u_{2, t} u_{3, t}\right)^{\frac{1}{2}}+\left(1-\widetilde{u}_{2, t} \widetilde{u}_{3, t}\right)^{\frac{1}{2}}\right]=0, \\
& \left(u_{3, t}-\widetilde{u}_{3, t}\right)\left[p^{2}-\left(u_{2}+\widetilde{u}_{2}\right)\left(u_{3}+\widetilde{u}_{3}\right)\right]^{\frac{1}{2}}+\left(u_{3}+\widetilde{u}_{3}\right)\left[\left(1-u_{2, t} u_{3, t}\right)^{\frac{1}{2}}+\left(1-\widetilde{u}_{2, t} \widetilde{u}_{3, t}\right)^{\frac{1}{2}}\right]=0,
\end{aligned}
$$


where $u_{2}=u_{2}(n, t)$ and $u_{3}=u_{3}(n, t)$. In full continuum limit

$$
n \rightarrow \infty, p \rightarrow \infty, x=2 \frac{n}{p} \sim O(1),
$$

in which $x$ can be identified as the continuous space variable, we have

$$
\rho \rightarrow e^{\kappa x-2 \frac{t}{\kappa}} \rho^{0} .
$$

The equation (5.2) yields (in terms of $p^{0}$ and $\boldsymbol{u}=\boldsymbol{u}(x, t)$ )

$$
\begin{aligned}
& 2 \boldsymbol{u}_{x t}=(\boldsymbol{a} \boldsymbol{u}-\tilde{\boldsymbol{u}} \boldsymbol{a})\left(\boldsymbol{a}+\boldsymbol{u}_{t}\right)-\left(\boldsymbol{a}+\boldsymbol{u}_{t}\right)(\boldsymbol{a} \boldsymbol{u}-\tilde{\boldsymbol{u}} \boldsymbol{a}), \\
& \left(\boldsymbol{a}+\boldsymbol{u}_{t}\right)^{2}=\boldsymbol{I}_{2}, \\
& 2 \boldsymbol{a} \boldsymbol{u}_{x}+2 \boldsymbol{u}_{x} \boldsymbol{a}+(\boldsymbol{a} \boldsymbol{u}-\boldsymbol{u} \boldsymbol{a})^{2}=\mathbf{0} .
\end{aligned}
$$

Then the substitution of (3.17a) into (5.5) leads to the equation

$$
\begin{aligned}
& u_{2_{x t}}=-2 u_{2}-2 u_{2} \partial_{t} \partial^{-1}\left(u_{2} u_{3}\right), \\
& u_{3_{x t}}=-2 u_{3}-2 u_{3} \partial_{t} \partial^{-1}\left(u_{2} u_{3}\right),
\end{aligned}
$$

where $\partial=\frac{\partial}{\partial x}, \partial^{-1} \partial=\partial \partial^{-1}=1$. System (5.6) is the continuous nAKNS equation (cf. [30]).

The solutions for both semi-discrete nAKNS equation (5.3) and continuous nAKNS equation (5.6) are still expressed by (3.26) and possess four types of exact solutions given in Sec.4. The slight difference is that the plane wave factors (4.18) should be replaced by

$$
\begin{aligned}
\rho_{i} & =\left(\frac{p+k_{i}}{p-k_{i}}\right)^{n} e^{-2 \frac{t}{k_{i}}} \rho_{i}^{0}, \text { with constants } \rho_{i}^{0}, \\
\sigma_{j} & =\left(\frac{p-l_{j}}{p+l_{j}}\right)^{n} e^{2 \frac{t}{l_{j}}} \sigma_{j}^{0}, \text { with constants } \sigma_{j}^{0},
\end{aligned}
$$

for semi-discrete case and

$$
\begin{aligned}
& \rho_{i}=e^{k_{i} x-2 \frac{t}{k_{i}}} \rho_{i}^{0}, \text { with constants } \rho_{i}^{0}, \\
& \sigma_{j}=e^{-l_{j} x+2 \frac{t}{l_{j}}} \sigma_{j}^{0}, \text { with constants } \sigma_{j}^{0},
\end{aligned}
$$

for continuous case.

\section{Reduction}

In this section, by imposing constraint on matrices $\boldsymbol{K}_{1}$ and $\boldsymbol{K}_{2}$ in determining equation set (2.2) and (3.1), we discuss the reduction of resulting discrete nAKNS equation (3.25). As a consequence, 
discrete SG equation is obtained. To start, we take $N_{1}=N_{2}=N$ and $\boldsymbol{K}_{2}=-\boldsymbol{K}_{1}$. Then (2.4) becomes

$$
\begin{aligned}
& \boldsymbol{K}_{1} \boldsymbol{M}_{1}+\boldsymbol{M}_{1} \boldsymbol{K}_{1}=\boldsymbol{r}_{1}{ }^{t} \boldsymbol{c}_{2}, \\
& -\boldsymbol{K}_{1} \boldsymbol{M}_{2}-\boldsymbol{M}_{2} \boldsymbol{K}_{1}=\boldsymbol{r}_{2}{ }^{t} \boldsymbol{c}_{1},
\end{aligned}
$$

and (3.1) yields

$$
\begin{aligned}
& \left(\begin{array}{cc}
p \boldsymbol{I}-\boldsymbol{K}_{1} & \mathbf{0} \\
\mathbf{0} & p \boldsymbol{I}-\boldsymbol{K}_{1}
\end{array}\right) \widetilde{\boldsymbol{r}}=\left(\begin{array}{cc}
p \boldsymbol{I}+\boldsymbol{K}_{1} & \mathbf{0} \\
\mathbf{0} & p \boldsymbol{I}+\boldsymbol{K}_{1}
\end{array}\right) \boldsymbol{r}, \\
& \left(\begin{array}{cc}
q \boldsymbol{I}-\boldsymbol{K}_{1}^{-1} & \mathbf{0} \\
\mathbf{0} & q \boldsymbol{I}-\boldsymbol{K}_{1}^{-1}
\end{array}\right) \hat{\boldsymbol{r}}=\left(\begin{array}{cc}
q \boldsymbol{I}+\boldsymbol{K}_{1}^{-1} & \mathbf{0} \\
\mathbf{0} & q \boldsymbol{I}+\boldsymbol{K}_{1}^{-1}
\end{array}\right) \boldsymbol{r} .
\end{aligned}
$$

By the form of (6.2), we assume $\boldsymbol{r}_{1}=\boldsymbol{r}_{2}$. Meanwhile, we suppose ${ }^{t} \boldsymbol{c}_{1}={ }^{t} \boldsymbol{c}_{2}$. Then (6.1) implies $\boldsymbol{M}_{2}=-\boldsymbol{M}_{1}$. In this situation, from (2.10) we know that $u_{2}=u_{3}$. Consequently, both (3.25a) and (3.25b) reduce to a discrete $\mathrm{SG}$ equation

$$
\begin{aligned}
& q\left(\widehat{u}_{2}-u_{2}\right)\left[p^{2}-\left(u_{2}+\widetilde{u}_{2}\right)^{2}\right]^{\frac{1}{2}}+\left(u_{2}+\widetilde{u}_{2}\right)\left[1-q^{2}\left(\widehat{u}_{2}-u_{2}\right)^{2}\right]^{\frac{1}{2}} \\
& +\left(\widehat{u}_{2}+\widehat{\widetilde{u}}_{2}\right)\left[1-q^{2}\left(\widehat{\widetilde{u}}_{2}-\widetilde{u}_{2}\right)^{2}\right]^{\frac{1}{2}}+q\left(\widetilde{u}_{2}-\widehat{\widetilde{u}}_{2}\right)\left[p^{2}-\left(\widehat{u}_{2}+\widehat{\widetilde{u}}_{2}\right)^{2}\right]^{\frac{1}{2}}=0,
\end{aligned}
$$

which first appeared in [24]. The solution is

$$
u_{2}={ }^{t} \boldsymbol{c}_{1}\left(\boldsymbol{I}_{N}+\boldsymbol{M}_{1}^{2}\right)^{-1} \boldsymbol{r}_{1},
$$

where ${ }^{t} \boldsymbol{c}_{1}, \boldsymbol{M}_{1}$ and $\boldsymbol{r}_{1}$ are given by Sec. 4. Similarly, with constraint $u_{2}=u_{3}$, (5.3) leads to a semidiscrete $\mathrm{SG}$ equation

$$
\left(u_{2, t}-\widetilde{u}_{2, t}\right)\left[p^{2}-\left(u_{2}+\widetilde{u}_{2}\right)^{2}\right]^{\frac{1}{2}}+\left(u_{2}+\widetilde{u}_{2}\right)\left[\left(1-u_{2, t}^{2}\right)^{\frac{1}{2}}+\left(1-\widetilde{u}_{2, t}^{2}\right)^{\frac{1}{2}}\right]=0,
$$

and (5.6) reduces to continuous SG equation

$$
u_{2_{x t}}=-2 u_{2}-2 u_{2} \partial_{t} \partial^{-1}\left(u_{2}^{2}\right),
$$

where solution $u_{2}$ is given by (6.4) and plane wave factors are, respectively, replaced by (5.7a) and (5.8a).

Eq. (6.6) can be transformed into the usual continuous SG equation. In fact, multiplying $u_{2_{t}}$ on both sides of (6.6) and by integration $\partial^{-1}$, then by direct calculation we get

$$
1+\partial^{-1}\left(u_{2}^{2}\right)_{t}=\left(1-\left(u_{2_{t}}\right)^{2}\right)^{\frac{1}{2}} .
$$

Thus (6.6) can be rewritten as

$$
u_{2_{x t}}=-2 u_{2}\left(1-\left(u_{2_{t}}\right)^{2}\right)^{\frac{1}{2}} .
$$

With transformation $\phi=\arcsin u_{2_{t}},(6.8)$ gives rise to

$$
\phi_{x t}=-2 \sin \phi,
$$

which is the usual continuous SG equation. The solution for (6.9) reads

$$
\phi=\arcsin \left({ }^{t} \boldsymbol{c}_{1}\left(\boldsymbol{I}_{N}+\boldsymbol{M}_{1}^{2}\right)^{-1} \boldsymbol{r}_{1}\right)_{t},
$$

where ${ }^{t} \boldsymbol{c}_{1}, \boldsymbol{M}_{1}$ and $\boldsymbol{r}_{1}$ are given by Sec.4 and plane wave factor is defined by (5.8a). 


\section{Conclusions}

We study a discrete version of the nAKNS equation via generalized Cauchy matrix approach. The Sylvester equation of our interest is (2.2) and it defines the master function $\boldsymbol{S}^{(i, j)}$ (2.6). After imposing dispersion relation on $\boldsymbol{r}$, we get shifts (along with $n$-, $m$-directions) of $\boldsymbol{S}^{(i, j)}$. Then we derive a discrete nAKNS equation in closed form. Multi-soliton solutions, multiple-pole solutions, the simplest and the most general mixed solutions of the Jordan canonical determining equation set (4.5) and (6.2) are obtained by taking different forms of $\boldsymbol{\Gamma}$ and $\boldsymbol{\Lambda}$. The continuum limits of the discrete nAKNS equation are also considered and the solutions are discussed. Besides, the reduction to discrete, semi-discrete and continuous SG equations are also considered. The dynamical properties of the solutions for the resulting discrete and semi-discrete equations are worthy of consideration and left for further discussion. We are also interested in the discrete and semi-discrete versions of positive AKNS type equations (AKNS equation, modified AKNS equation and Schwarzian AKNS equation) and their exact solutions, which will be considered in the near future.

\section{Acknowledgments}

The author is grateful to the referee for the invaluable comments. This project is supported by the National Natural Science Foundation Grant (Nos.11301483, 11401529).

\section{References}

[1] M.J. Ablowitz and F.J. Ladik, A nonlinear difference scheme and inverse scattering, Stud. Appl. Math. 55 (1976) 213-229.

[2] M.J. Ablowitz and F.J. Ladik, On the solution of a class of nonlinear partial difference equations, Stud. Appl. Math. 57 (1977) 1-12.

[3] V.E. Adler, A.I. Bobenko and Yu.B. Suris, Classification of integrable equations on quad-graphs, the consistency approach, Commun. Math. Phys. 233 (2002) 513-543.

[4] V.E. Adler, A.I. Bobenko and Yu.B. Suris, Classification of integrable discrete equations of octahedron type, Int. Math. Res. Notices 2011 (2011) (68pp).

[5] J. Atkinson, J. Hietarinta and F. Nijhoff, Soliton solutions for Q3, J. Phys. A: Math. Theor. 41 (2008) 142001(11pp).

[6] A.I. Bobenko and Yu. B. Suris, Integrable systems on quad-graphs, Int. Math. Res. Notices 11 (2002) 573-611.

[7] S. Butler and N. Joshi, An inverse scattering transform for the lattice potential KdV equation, Inver. Prob. 26 (2010) 115012(28pp).

[8] R. Camassa and D.D. Holm, An integrable shallow-water equation with peakon solitons, Phys. Rev. Lett. 71 (1993) 1661-1664.

[9] C.W. Cao and X.X. Xu, A finite genus solution of the H1 model, J. Phys. A: Math. Theor. 45 (2012) 055213(13pp).

[10] E. Date, M. Jimbo and T. Miwa, Method for generating discrete soliton equations I-V, J. Phys. Soc. Japan 51 (1982) 4116-4131, 52 (1983) 388-393, 761-771.

[11] A. Degasperis, D.D. Holm and A.N.W. Hone, A new integrable equation with peakon solutions, Theor. Math. Phys. 133 (2002) 1463-1474.

[12] B. Fuchssteiner and A.S. Fokas, Symplectic structures, their Bäcklund transformations and hereditary symmetries, Phys. D 4 (1981) 47-66.

[13] J. Hietarinta, Boussinesq-like multi-component lattice equations and multi-dimensional consistency, $J$. Phys. A: Math. Theor. 44 (2011) 165204(22pp).

[14] J. Hietarinta and D.J. Zhang, Soliton solutions for ABS lattice equations: II. Casoratians and bilinearization, J. Phys. A: Math. Theor. 42 (2009) 404006(30pp). 
[15] J. Hietarinta and D.J. Zhang, Soliton taxonomy for a modification of the lattice Boussinesq equation, SIGMA 7 (2011) 061(14pp).

[16] R. Hirota, Nonlinear partial difference equations I-III, J. Phys. Soc. Japan 43 (1977) 1424-1433, 2074 2089.

[17] Y.S. Li and X.D. Ji, Integrable soliton equations and reciprocal transformation, preprint, (2007).

[18] F.W. Nijhoff, J. Atkinson and J. Hietarinta, Soliton solutions for ABS lattice equations: I. Cauchy matrix approach, J. Phys. A: Math. Theor. 42 (2009) 404005(34pp).

[19] F.W. Nijhoff, G.R.W. Quispel and H.W. Capel, Direct linearization of nonlinear difference-difference equations, Phys. Lett. 97A (1983) 125-128.

[20] F.W. Nijhoff and A.J. Walker, The discrete and continuous Painlevé VI hierarchy and the Garnier systems, Glasgow Math. J. 43A (2001) 109-123.

[21] J.J.C. Nimmo, Darboux transformations and the discrete KP equation, J. Phys. A: Math. Gen. 30 (1997) 8693-8704.

[22] Z.J. Qiao and E.G. Fan, Negative-order Korteweg-de Vries equations, Phys. Rev. E 86 (2012) 016601(20pp).

[23] Z.J. Qiao and W. Strammp, Negative order mKdV hierarchy and a new integrable Neumann-like system, Phys. A 313 (2002) 365-380.

[24] G.R.W. Quispel, F.W. Nijhoff, H.W. Capel and J. van der Linden, Linear integral equations and nonlinear difference-difference equations, Phys. A 125 (1984) 344-380.

[25] T. Schäfter and C.E. Wayne, Propagation of ultra-short optical pulses in cubic nonlinear media, Phys. D 196 (2004) 90-105.

[26] J. Sylvester, Sur l'equation en matrices $p x=x q$, C. R. Acad. Sci. Paris 99 (1884) 67-71, 115-116.

[27] D.D. Xu, D.J. Zhang and S.L. Zhao, The Sylvester equation and integrable equations: I. The Kortewegde Vries system and sine-Gordon equation, J. Nonlin. Math. Phys. 21(3) (2014) 382-406.

[28] G.F. Yu, Discrete analogues of a negative order AKNS equation, Stud. Appl. Math. 135 (2015) 117-138.

[29] D.J. Zhang, Notes on solutions in Wronskian form to soliton equations: KdV-type, arXiv:nlin.SI/0603008 (2006).

[30] D.J. Zhang, J. Ji and S.L. Zhao, Soliton scattering with amplitude changes of a negative order AKNS equation, Phys. D 238 (2009) 2361-2367.

[31] D.J. Zhang and S.L. Zhao, Solutions to ABS lattice equations via generalized Cauchy matrix approach, Stud. Appl. Math. 131 (2013) 72-103.

[32] D.J. Zhang, S.L. Zhao and F.W. Nijhoff, Direct Linearization of extended lattice BSQ systems, Stud. Appl. Math. 129 (2012) 220-248.

\section{Appendix A. Inverse of block matrix}

Here we consider the inverse of the block matrix. We describe this in the following proposition

Proposition Appendix A.1. For the block matrix $\boldsymbol{\Phi}=\left(\begin{array}{ll}\boldsymbol{A} & \boldsymbol{B} \\ \boldsymbol{C} & \boldsymbol{D}\end{array}\right)$, where $\boldsymbol{A}$ and $\boldsymbol{D}$ are, respectively, $N_{1} \times N_{1}$ and $N_{2} \times N_{2}$ invertible matrices, and $\boldsymbol{B}$ and $\boldsymbol{C}$ are, respectively, $N_{1} \times N_{2}$ and $N_{2} \times N_{1}$ matrices. Then the inverse matrix of $\boldsymbol{\Phi}$ can be described as

$$
\boldsymbol{\Phi}^{-1}=\left(\begin{array}{cc}
\left(\boldsymbol{A}-\boldsymbol{B} \boldsymbol{D}^{-1} \boldsymbol{C}\right)^{-1} & -\boldsymbol{A}^{-1} \boldsymbol{B}\left(\boldsymbol{D}-\boldsymbol{C A}^{-1} \boldsymbol{B}\right)^{-1} \\
-\boldsymbol{D}^{-1} \boldsymbol{C}\left(\boldsymbol{A}-\boldsymbol{B} \boldsymbol{D}^{-1} \boldsymbol{C}\right)^{-1} & \left(\boldsymbol{D}-\boldsymbol{C A}^{-1} \boldsymbol{B}\right)^{-1}
\end{array}\right)
$$

Proof. By elementary transformation, we have

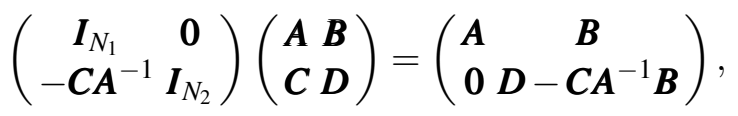


then

$$
\begin{aligned}
& \boldsymbol{\Phi}^{-1}=\left(\begin{array}{ll}
\boldsymbol{A} & \boldsymbol{B} \\
\mathbf{0} & \boldsymbol{D}-\boldsymbol{C A}^{-1} \boldsymbol{B}
\end{array}\right)^{-1}\left(\begin{array}{cc}
\boldsymbol{I}_{N_{1}} & \mathbf{0} \\
-\boldsymbol{C A}^{-1} & \boldsymbol{I}_{N_{2}}
\end{array}\right) \\
& =\left(\begin{array}{cc}
\boldsymbol{A}^{-1} & -\boldsymbol{A}^{-1} \boldsymbol{B}\left(\boldsymbol{D}-\boldsymbol{C A}^{-1} \boldsymbol{B}\right)^{-1} \\
\mathbf{0} & \left(\boldsymbol{D}-\boldsymbol{C A}^{-1} \boldsymbol{B}\right)^{-1}
\end{array}\right)\left(\begin{array}{cc}
\boldsymbol{I}_{N_{1}} & \mathbf{0} \\
-\boldsymbol{C A}^{-1} & \boldsymbol{I}_{N_{2}}
\end{array}\right) \\
& =\left(\begin{array}{cc}
\boldsymbol{A}^{-1}+\boldsymbol{A}^{-1} \boldsymbol{B}\left(\boldsymbol{D}-\boldsymbol{C A}^{-1} \boldsymbol{B}\right)^{-1} \boldsymbol{C} \boldsymbol{A}^{-1}-\boldsymbol{A}^{-1} \boldsymbol{B}\left(\boldsymbol{D}-\boldsymbol{C A}^{-1} \boldsymbol{B}\right)^{-1} \\
-\left(\boldsymbol{D}-\boldsymbol{C} \boldsymbol{A}^{-1} \boldsymbol{B}\right)^{-1} \boldsymbol{C} \boldsymbol{A}^{-1} & \left(\boldsymbol{D}-\boldsymbol{C} \boldsymbol{A}^{-1} \boldsymbol{B}\right)^{-1}
\end{array}\right) .
\end{aligned}
$$

By elementary transformation, we have

$$
\left(\begin{array}{cc}
\boldsymbol{I}_{N_{1}} & -\boldsymbol{B} \boldsymbol{D}^{-1} \\
\mathbf{0} & \boldsymbol{I}_{N_{2}}
\end{array}\right)\left(\begin{array}{ll}
\boldsymbol{A} & \boldsymbol{B} \\
\boldsymbol{C} & \boldsymbol{D}
\end{array}\right)=\left(\begin{array}{cr}
\boldsymbol{A}-\boldsymbol{B} \boldsymbol{D}^{-1} \boldsymbol{C} & \mathbf{0} \\
\boldsymbol{C} & \boldsymbol{D}
\end{array}\right)
$$

then

$$
\begin{aligned}
\boldsymbol{\Phi}^{-1} & =\left(\begin{array}{cc}
\boldsymbol{A}-\boldsymbol{B} \boldsymbol{D}^{-1} \boldsymbol{C} & \mathbf{0} \\
\boldsymbol{C} & \boldsymbol{D}
\end{array}\right)^{-1}\left(\begin{array}{cc}
\boldsymbol{I}_{N_{1}} & -\boldsymbol{B} \boldsymbol{D}^{-1} \\
\mathbf{0} & \boldsymbol{I}_{N_{2}}
\end{array}\right) \\
& =\left(\begin{array}{cc}
\left(\boldsymbol{A}-\boldsymbol{B} \boldsymbol{D}^{-1} \boldsymbol{C}\right)^{-1} & \mathbf{0} \\
-\boldsymbol{D}^{-1} \boldsymbol{C}\left(\boldsymbol{A}-\boldsymbol{B} \boldsymbol{D}^{-1} \boldsymbol{C}\right)^{-1} & \boldsymbol{D}^{-1}
\end{array}\right)\left(\begin{array}{cc}
\boldsymbol{I}_{N_{1}}-\boldsymbol{B} \boldsymbol{D}^{-1} \\
\mathbf{0} & \boldsymbol{I}_{N_{2}}
\end{array}\right) \\
& =\left(\begin{array}{cc}
\left(\boldsymbol{A}-\boldsymbol{B} \boldsymbol{D}^{-1} \boldsymbol{C}\right)^{-1} & -\left(\boldsymbol{A}-\boldsymbol{B} \boldsymbol{D}^{-1} \boldsymbol{C}\right)^{-1} \boldsymbol{B} \boldsymbol{D}^{-1} \\
-\boldsymbol{D}^{-1} \boldsymbol{C}\left(\boldsymbol{A}-\boldsymbol{B} \boldsymbol{D}^{-1} \boldsymbol{C}\right)^{-1} & \boldsymbol{D}^{-1}+\boldsymbol{D}^{-1} \boldsymbol{C}\left(\boldsymbol{A}-\boldsymbol{B} \boldsymbol{D}^{-1} \boldsymbol{C}\right)^{-1} \boldsymbol{B} \boldsymbol{D}^{-1}
\end{array}\right) .
\end{aligned}
$$

In terms of the uniqueness of the inverse, we have

$$
\boldsymbol{\Phi}^{-1}=\left(\begin{array}{cc}
\left(\boldsymbol{A}-\boldsymbol{B} \boldsymbol{D}^{-1} \boldsymbol{C}\right)^{-1} & -\boldsymbol{A}^{-1} \boldsymbol{B}\left(\boldsymbol{D}-\boldsymbol{C A}^{-1} \boldsymbol{B}\right)^{-1} \\
-\boldsymbol{D}^{-1} \boldsymbol{C}\left(\boldsymbol{A}-\boldsymbol{B} \boldsymbol{D}^{-1} \boldsymbol{C}\right)^{-1} & \left(\boldsymbol{D}-\boldsymbol{C} \boldsymbol{A}^{-1} \boldsymbol{B}\right)^{-1}
\end{array}\right) .
$$

